\title{
Phase diagram for diblock copolymer melts under cylindrical confinement
}

\author{
Weihua Li and Robert A. Wickham \\ Department of Physics, St. Francis Xavier University, \\ Antigonish, Nova Scotia, Canada B2G 2W5
}

(Dated: October 18, 2018)

\begin{abstract}
We extensively study the phase diagram of a diblock copolymer melt confined in a cylindrical nanopore using real-space self-consistent mean-field theory. We discover a rich variety of new twodimensional equilibrium structures that have no analog in the unconfined system. These include non-hexagonally coordinated cylinder phases and structures intermediate between lamellae and cylinders. We map the stability regions and phase boundaries for all the structures we find. As the pore radius is decreased, the pore accommodates fewer cylindrical domains and structural transitions occur as cylinders are eliminated. Our results are consistent with experiments, but we also predict phases yet to be observed.

PACS numbers: 82.35.Jk, 61.41.+e, 61.46.+w
\end{abstract}


Self-assembly in macromolecular systems provides a convenient route to create structure at the nanoscale. These structures have potential applications as, for example, lithographic templates for nanowires, photonic crystals, and high-density magnetic storage media [1]. Confinement effects, produced by boundaries, influence the self-assembly process and can generate novel nanostructures. In a rational search for new morphologies to fit a particular application, it is crucial to know which structures are possible in a confined system, and under what conditions, i.e. one needs to know the phase diagram.

Confined diblock copolymer melts have become the focus of increasing attention. In the bulk, these materials self-assemble into a variety of periodic nanostructures - lamellae, hexagonally-coordinated cylinders, body-centred cubic lattices of spheres, and the gyroid morphology. The ability to tune the period and to control self-assembly using temperature, chemical composition, and molecular architecture, lend a rich physics to these materials, and make them attractive for industrial applications [1, 2, 3, 4]. Competition between the chain stretching energy and the interfacial energy between block domains determines which structures form in the bulk. Introducing confinement modifies this competition. Confinement of diblock copolymers between parallel solid walls, or in a thin film, has been extensively studied [5, 6, 7, 8, 9]. However, the effect of confinement in a cylindrical pore is relatively unexplored.

Recently, Xiang et al. examined asymmetric and symmetric polystyrene- $b$-polybutadiene (PS- $b$-PBD) diblock copolymers confined to cylindrical alumina nanopores and observed cylindrical and concentric lamellar structures in the pores [10]. Concentric lamellar structures were also seen in the experiments of Sun et al. involving symmetric polystyrene- $b$-poly(methyl methacrylate) (PS- $b$-PMMA) diblock copolymers confined in alumina nanopores 11]. Concentric lamellar structures have been seen in the Monte Carlo simulations of He et al. [12] and in the dynamical density functional simulations of Sevink et al. 13]. Experiments using narrower pores find that stacked-disc or toroidal-type structures, and also cylindrical helices, are possible [14, 15]. Such three-dimensional structures have been seen in the simulations of Ref. 13] and in a self-consistent mean-field theory (SCMFT) study of self-assembled silica-surfactant mesostructures in cylindrical nanopores 16]. However, in all the simulation and theoretical work to date, only a few phases, in a very limited region of parameter space, have been studied due to the time and computational requirements. Knowledge of the phase behaviour of cylindrically confined block copolymers 
is thus currently fragmentary.

To fill this knowledge gap, we systematically evaluate the phase diagram for a diblock copolymer melt confined in a cylindrical nanopore using SCMFT. SCMFT has proved to be one of the most successful theoretical methods for investigating equilibrium phases in block copolymers, and has played a major role in establishing the phase diagram of the bulk diblock copolymer melt [17, 18]. We explore the phase diagram for the cylindrically confined melt, and identify the equilibrium phase boundaries and stability regions for various phases that exist in this system. We consider structures that are translationally-invariant along the pore axis, and are thus two-dimensional. Previous works have examined on the order of 10 points in the phase diagram. Our study examines approximately 3000 points in the phase diagram and consumes approximately 15,000 cpu-hours of a $3 \mathrm{GHz}$ Intel Xeon processor.

We consider an incompressible melt of $\mathrm{AB}$ diblock copolymers, confined to a cylindrical pore of radius $R$. Each copolymer has a degree of polymerization $N$ while the A-block on each has a degree of polymerization $f N$ with $0 \leq f \leq 1$. Lengths in our theory are expressed in units of the radius of gyration, $R_{a}$, of the polymer. Within the mean-field approximation to the many-chain Edwards theory [19, 20], at a temperature $T$ the free-energy $F$ per chain for $n$ Gaussian diblock copolymer chains confined in a cylindrical pore has the form

$$
\begin{array}{r}
\frac{F}{n k_{B} T}=-\ln Q+\frac{1}{V} \int_{|\mathbf{r}| \leq R} d \mathbf{r}\left\{\chi N \phi_{A}(\mathbf{r}) \phi_{B}(\mathbf{r})-\omega_{A}(\mathbf{r}) \phi_{A}(\mathbf{r})-\omega_{B}(\mathbf{r}) \phi_{B}(\mathbf{r})\right. \\
\left.+H(\mathbf{r})\left[\phi_{A}(\mathbf{r})-\phi_{B}(\mathbf{r})\right]\right\} .
\end{array}
$$

The monomer densities are $\phi_{A}$ and $\phi_{B}$, the partition function $Q$ is for a single polymer interacting with the mean-fields $\omega_{A}$ and $\omega_{B}$ produced by the surrounding chains. These quantities have the standard definitions and meanings [17, 18]. The Flory-Huggins interaction parameter, $\chi$, characterizes the repulsion between dissimilar monomers. In the confined melt, the spatial integration is restricted to the cylinder volume, taken to be $V$. We assume the pore wall has a preference for B monomers and include a surface field $H(\mathbf{r})$ in Eq. (11) that attracts species B. For convenience, this surface field is chosen to have the form

$$
\frac{H(\mathbf{r})}{\chi N}=V_{0}\{\exp [(\sigma+|\mathbf{r}|-R) / \lambda]-1\}
$$

for $R-\sigma \leq|\mathbf{r}| \leq R$, while $H(\mathbf{r})=0$ for $|\mathbf{r}|<R-\sigma$. We choose the cutoff distance for the surface interaction to be $\sigma=0.4 R_{g}$, and the decay length to be $\lambda=0.2 R_{g}$. We fix the strength of the surface field to be $V_{0}=0.4$. We find that modest variations in $V_{0}$ have little effect on the phase diagram and morphologies. 
Minimization of the free-energy with respect to the monomer densities and mean-fields leads to a set of mean-field equations which can be solved self-consistently in real space to find the equilibrium densities [17, 18, 21]. We employ the split-step Fourier method of Tzeremes et al. 22] to solve the modified diffusion equations for the end-segment distribution functions 17] on a $128 \times 128$ square lattice, with a lattice constant of $0.136 R_{g}$. The chain contour length for each block is discretized into 128 segments. We find that the stability regions and phase boundaries are not significantly altered if we use a finer mesh, indicating that a $128 \times 128$ lattice provides sufficient accuracy [23]. Incompressibility, $\phi_{A}(\mathbf{r})+\phi_{B}(\mathbf{r})=1$, is enforced via a Lagrange multiplier for $|\mathbf{r}|<R$. Outside the pore, $|\mathbf{r}| \geq R$, there is no polymer, so we set the end-segment distribution functions to zero outside the pore, which implies that $\phi_{A}=\phi_{B}=0$ in this region. Other than assuming that the structures are translation-invariant along the axis of the pore, we do not make any a priori assumptions about the symmetry of potential equilibrium structures. This is a major advantage of the real-space approach [21]. We first use random initial conditions in our iterative algorithm to generate a large set of solutions to the mean-field equations over a wide region of phase space. Once we determine this set, we then use these solutions as initial conditions in our algorithm, to explore the extent (if any) of their stability regions. We take the equilibrium phase to be the structure which has the lowest free-energy, for a given $f$ and $\chi N$, of all the observed structures.

We first examine the phases that form in a fixed pore radius, $R=8.5 R_{g}$, and later discuss results for different radii. The 21 equilibrium nanostructured phases found at this pore radius are shown in Fig. 1. The notation we use for the structures is explained in the caption of Fig. 1] The phase diagram is presented in Fig. 2, and has some features in common with the phase diagram for the unconfined diblock copolymer melt [17]. The disordered (D) phase is stable when $\chi N$ is low, or when the copolymer is close to being a homopolymer $(f \rightarrow 0$ or 1$)$. Above the order-disorder transition, for $f \lesssim 0.3$, the asymmetry in the block composition favours curved interfaces and the formation of cylindrical domains of the minority A species. Inverted cylindrical domains, composed of the minority B species, form when $f \gtrsim 0.65$. Symmetric copolymers favour $\mathrm{AB}$ interfaces with low curvature and thus concentric lamellar rings form in the centre of the phase diagram. There are, however, significant differences between our phase diagram and the phase diagram for the bulk system, which we now discuss. 
Our phase diagram is much more intricate than the phase diagram for the unconfined melt. Many of the trends in the phase diagram can be understood in terms of packing a finite number of domains into the pore, which has a finite cross-sectional area. For a given $R, f$ and $\chi N$, there is an optimum number of domains that can be accommodated - this number minimizes the free-energy penalties associated with chain stretching or compression. At fixed $R$, as $\chi N$ increases, we see from Figs. 1 and 2 that the domain size increases, and the number of domains in the pore decreases. For example, as $\chi N$ increases at fixed $f=0.22$ the structure changes from $C_{1-6-13}$ to $C_{1-6-11}$ to $C_{1-5-11}$ to $C_{4-10}$. A similar trend is seen for the inverted cylinder phases, and for the concentric lamellae. For the cylinder phases of minority A (B) species, a similar argument explains the trends with increasing (decreasing) $f$. These packing considerations are purely the result of confinement, and do not arise in the bulk.

We observe arrangements of cylinders in the inner region of the pore with non-hexagonal coordination, such as the $C_{4-10}, C_{1-5-11}$ an $\bar{C}_{1-7}$ phases. Cylindrical structures with nonhexagonal coordination are not seen as equilibrium phases in the bulk, since such structures produce a highly non-uniform majority domain thickness, with an associated free-energy penalty [18]. Our observation of non-hexagonally coordinated cylinders suggests that, when confined, the system will tolerate a non-uniform majority domain thickness if it can avoid the compression (or stretching) penalty of packing a non-optimum number of cylinders into the pore.

For a narrow region $0.3 \lesssim f \lesssim 0.4$ in Fig. 2 we see structures that are intermediate between cylinders and lamellae. As in the unconfined melt, a value of $f$ closer to 0.5 favours $\mathrm{AB}$ interfaces of lower curvature. For example, for $\chi N=23$, as $f$ increases above $f \approx 0.3$ the $C_{4-10}$ structure will transform into $L C_{4}$ as the presence of the pore wall first leads the outer ring of cylinders to coalesce into a single narrow domain of the A phase. With increasing $f$, the AB interfacial curvature is lowered further as cylinders coalesce into lamellar segments in the inner region and the system transforms to the $L C_{L, 2}$ and then $L C_{L L}$ phases. When $f$ increases above $f \approx 0.4$ the high curvature of the semi-circular end-caps on the lamellar segments becomes unfavorable, leading to the formation of concentric lamellar rings. Like the gyroid phase in the unconfined diblock copolymer melt, these intermediate phases represent a compromise between pure cylindrical and pure lamellar order.

There is an asymmetry in the phase diagram, arising from the preference of the pore 
wall for the B block. In Figs. 1 and 2 the arrangements of the inverted cylinders and the stability regions for these structures are less complicated than the cylindrical structures seen for $f \lesssim 0.3$. One can think about the behaviour in this region in terms of the behaviour for $f \lesssim 0.3$ by exchanging the $A$ and $B$ monomers, and recognizing that the inverted cylinders exist in a pore with a smaller effective radius. In the inverted cylinder phases, a thick double layer of A monomers exists between the thin layer of minority B block along the pore wall and the cylinders in the inner region. We suggest that the outer A block, attached to the B block along the actual pore wall, acts as filler, creating an effective pore wall (of smaller radius) with a preference for $\mathrm{A}$. As we see below, when the radius of the pore decreases, fewer cylinders form in the pore.

All the order-order transitions we observe are first-order phase transitions. As the orderdisorder transition (ODT) is approached the inner region can disorder first (the $C_{1-6-13}$ to $C_{0-0-13}$ transition, for example) before the final transition to disorder. The ODT is first order, except perhaps for the ODT from $L_{3}$ to $D$. We are unable to determine the location of the ODT from $L_{3}$ to $D$, and the dashed curve shown in this region in Fig. 2 is an interpolation of the full ODT curve. As the $L_{3}$ to $D$ transition is approached along the ODT, from high or low $f$, the first order ODT becomes weaker. We speculate that the $L_{3}$ to $D$ transition may be continuous or, possibly, a crossover.

We explore the effect of changing the pore radius $R$ for the point $f=0.26$ and $\chi N=$ 20. Our results are shown in Fig. 3. With increasing pore radius, the complexity of the structures, both in terms of number of cylinders and the number of rings, increases. We observe the effect of commensurability of the pore radius with the hexagonal arrangement of cylinders expected in the bulk - the $C_{1-6}$ phase appears in smaller pores and then, following intervening, non-hexagonal phases, reappears as the $C_{1-6-12}$ phase in larger pores. Our expectation is that, for larger pore sizes, a hexagonal array of cylinders will preferentially form in the inner region of the pore in order to minimize unfavourable non-uniformities in the majority domain thickness. The $C_{1-6}, C_{1-7}$ and $C_{1-8}$ phases we observe here correspond, when $\mathrm{A}$ is exchanged with $\mathrm{B}$, to structures seen in Fig. 2 for $f \gtrsim 0.65$, supporting the idea that these latter structures form due to confinement in a pore with a smaller effective size.

Experiments by Xiang et al. examined both symmetric and asymmetric PS- $b$-PBD diblock copolymer melts confined to cylindrical nanopores in an alumina membrane [10]. The PBD block preferentially segregated to the pore wall and can therefore be identified as the 
B block. Using an asymmetric copolymer, with a PS volume fraction of 0.64 , they observed a structure similar to $\bar{C}_{1-6}$ in a pore of approximate diameter $140 \mathrm{~nm}$ (Fig. 4 of Ref. [10]) . Using a symmetric copolymer, with a PS volume fraction of 0.44 , they observed two concentric lamellar rings, similar to our $L_{2}$ structure, in an approximately $170 \mathrm{~nm}$ diameter pore (Fig. 6 of Ref. [10]). We estimate that $R_{g} \approx 7-8 \mathrm{~nm}$ in these experiments; therefore the 17 $R_{g}$ pore diameter used here is comparable the experimental pore diameter. The experiments of Sun et al., involving symmetric PS- $b$-PMMA $\left(R_{g} \approx 10 \mathrm{~nm}\right)$, also observe the $L_{2}$ phase in $180 \mathrm{~nm}$ diameter pores [11]. Structures with non-hexagonal symmetry, such as the ones we predict here, have not been observed experimentally, to our knowledge. Our work suggests that such structures exist for smaller values of $f$ or higher temperatures than used in experiments. Recent experiments suggest that three-dimensional structures can form in narrow pores (of radius 2-4 $R_{g}$ ) [14, 15]; furthermore, we expect spherical domains to form when the composition of the copolymer is highly asymmetric. We are currently investigating how our phase diagram is modified when three-dimensional structures are allowed in the theory.

To summarize, we have extensively explored the phase diagram of a diblock copolymer melt confined to a cylindrical nanopore through the use of real-space SCMFT. The phase diagram is more complicated than for the unconfined system, and we have found new twodimensional structures not seen in the bulk, notably non-hexagonally coordinated cylindrical phases and structures intermediate between lamellae and cylinders. A major achievement of our work is that our map of the phase stability regions and phase transition curves now enables thermodynamic issues for this system, such as the nature of transitions between different structures, to be explored. Many trends in the phase diagram can be understood in terms of the ability of the system to pack domains into the finite-radius pore. For example, we observe that with decreasing pore radius fewer cylinders can be accommodated. Our results are consistent with recent experimental observations, but we also predict a wealth of phases in parameter regions yet to be explored experimentally.

The authors gratefully acknowledge helpful discussions with Profs. A.-C. Shi, D. Hunter, and P. H. Poole, and with Dr. K. Rasmussen. The authors also thank the StFX hpcLAB and G. Lukeman for computing resources and support. This work was supported by NSERC, CFI and AIF. 
[1] C. Park, J. Yoon, and E. L. Thomas, Polymer 44, 6725 (2003).

[2] M. Park, C. Harrison, P. M. Chaikin, R. A. Register, and D. H. Adamson, Science 276, 1401 (1997).

[3] R. A. Segalman, H. Yokoyama, and E. J. Kramer, Adv. Mater. 13, 1152 (2001).

[4] J. Y. Cheng, A. M. Mayes, and C. A. Ross, Nature Mater. 3, 823 (2004).

[5] M. W. Matsen, J. Chem. Phys. 106, 7781 (1997).

[6] T. L. Morkved and H. M. Jaeger, Europhys. Lett. 40, 643 (1997).

[7] T. Geisinger, M. Müller, and K. Binder, J. Chem Phys. 111, 5241 (1999).

[8] H. P. Huinink, J. C. M. Brokken-Zijp, M. A. van Dijk, and G. J. A. Sevink, J. Chem Phys. 112, $2452(2000)$.

[9] K. Ø. Rasmussen, J. Polym. Sci. Part B: Polym. Phys. 42, 3695 (2004).

[10] H. Xiang et al. , Macromolecules 37, 5660 (2004).

[11] Y. Sun et al., Macromol. Rapid Commun. 26, 369 (2005).

[12] X. He, M. Song, H. Liang, and C. Pan, J. Chem. Phys. 114, 10510 (2001).

[13] G. J. A. Sevink, A. V. Zvelindovsky, J. G. E. M. Fraaije, and H. P. Huinink, J. Chem. Phys. 115, 8226 (2001).

[14] K. Shin et al. , Science 306, 76 (2004).

[15] H. Xiang et al. , Macromolecules 38, 1055 (2005).

[16] Y. Wu et al. , Nature Mater. 3, 816 (2004).

[17] M. W. Matsen and M. Schick, Phys. Rev. Lett. 72, 2660 (1994).

[18] M. W. Matsen, J. Phys.: Condens. Matter 14, R21 (2002).

[19] E. Helfand, J. Chem. Phys. 62, 999 (1975).

[20] K. M. Hong and J. Noolandi, Macromolecules 14, 727 (1981).

[21] F. Drolet and G. H. Fredrickson, Phys. Rev. Lett. 83, 4317 (1999).

[22] G. Tzeremes, K. Ø. Rasmussen, T. Lookman, and A. Saxena, Phys. Rev. E. 65, 041806-1 (2002).

[23] While the absolute free-energies of the structures are sensitive to the discretization used, the differences between free-energies are only slightly influenced by discretization. As a consequence, the phase boundaries are not significantly changed. 


\section{Figure Captions}

FIG. 1: Monomer density plots of the 21 nanostructured phases formed in the $8.5 R_{g}$ radius pore. The colour ranges from deep red (A-rich regions) to deep blue (B-rich regions). The region outside the cylindrical pore is also coloured deep blue. We use the notation $C$ for cylindrical phases, $L$ for lamellar phases, and $L C$ for structures containing both lamellae and cylinders, which we call "intermediate phases". When the cylinders are composed of minority B component an over-bar $(\bar{C}$ or $L \bar{C})$ is used. The number of cylinders in each ring, out from the centre of the pore, is indicated by subscripts $\mathrm{C}_{i-j-k}$. The number of $L$ subscripts in the notation $\mathrm{LC}_{L \cdots L, i-j}$ indicates the number lamellar segments in the inner region of a given intermediate structure. The second subscript indicates the number of cylinders of the minority A species in the pore, and whether these cylinders are arranged in rings. The stability regions for these structures are labelled on the phase diagram in Figure 2

FIG. 2: Phase diagram for a diblock copolymer melt confined in a cylindrical nanopore of radius $R=8.5 R_{g}$. The degree of polymerization of the copolymer is $N$, the Flory-Huggins parameter is $\chi$, and $f$ is the $A$ monomer fraction. The disordered phase is labelled $D$. The dashed curve is an interpolation of the order-disorder transition curve, as explained in the text.

FIG. 3: Phase stability regions as a function of pore radius. Colours indicate the stability range for each of the structures indicated, as the radius of the the cylindrical pore is increased from $5 R_{g}$ to $9 R_{g}$ at $f=0.26$ and $\chi N=20$. 

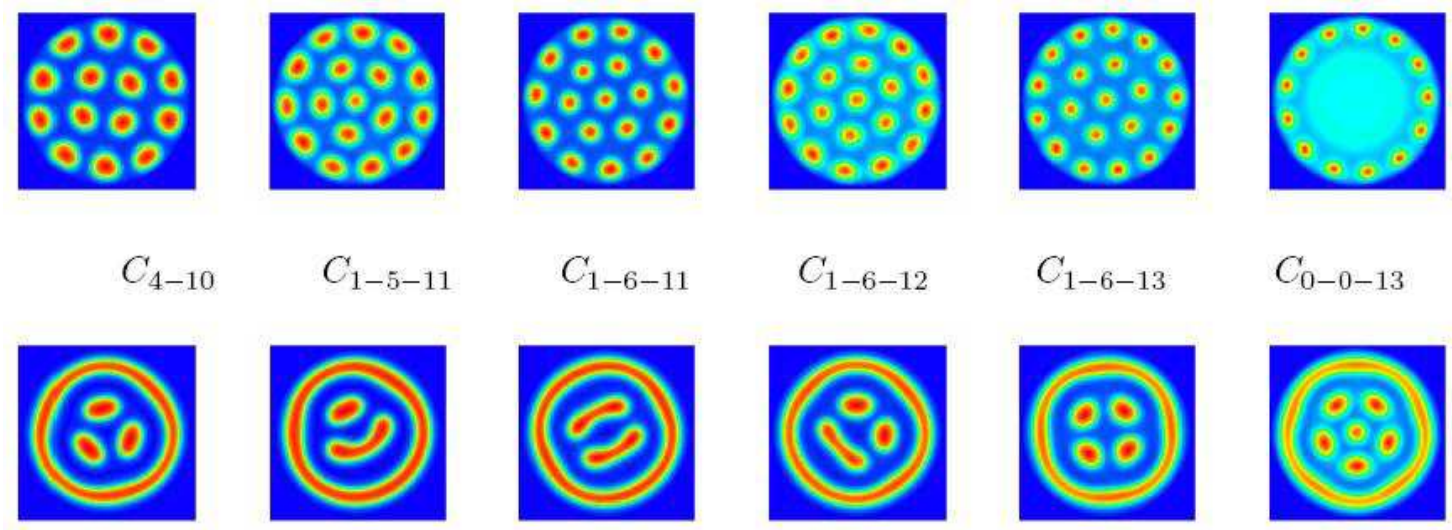

$$
C_{1-5-11}
$$

$C_{1-6-11}$

$C_{1-6-12}$

$C_{1-6-13}$

$C_{0-0-13}$
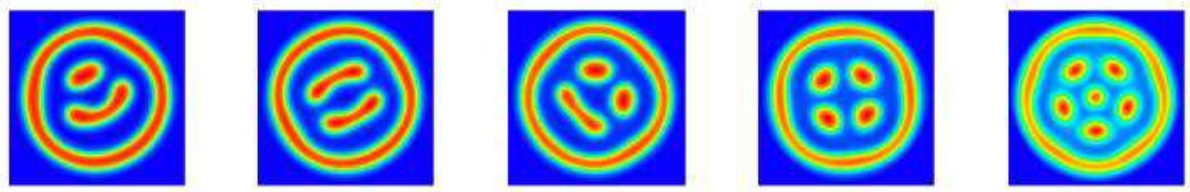

$a: L C_{3}$

$b: L C_{L, 1}$

$c: L C_{L L}$

$d: L C_{L, 2}$

$e: L C_{4}$

$L C_{1-5}$
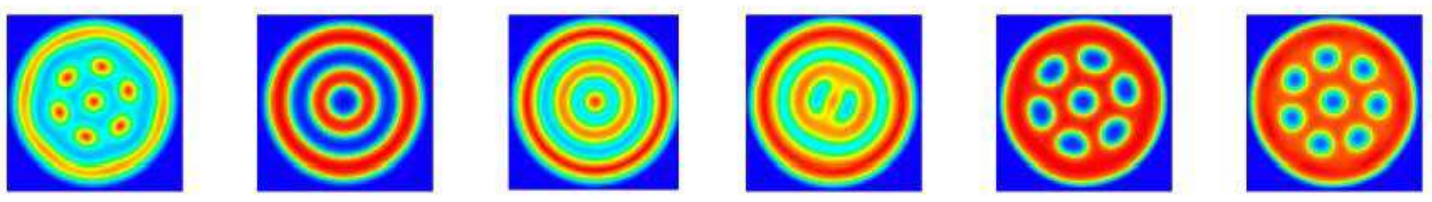

$$
L C_{1-6}
$$

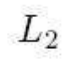

$L_{3}$

$L \bar{C}_{2}$

$\bar{C}_{1-6}$

$\bar{C}_{1-7}$
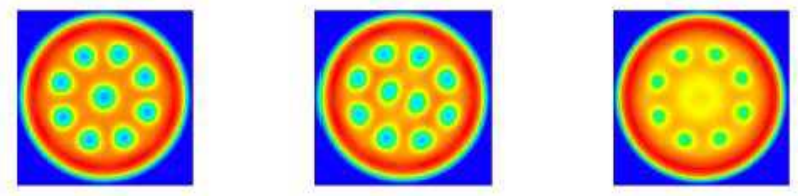
$\bar{C}_{1-8}$
$\bar{C}_{2-8}$
$\bar{C}_{0-8}$

Figure $1-$ Li 
Figure $2-$ Li

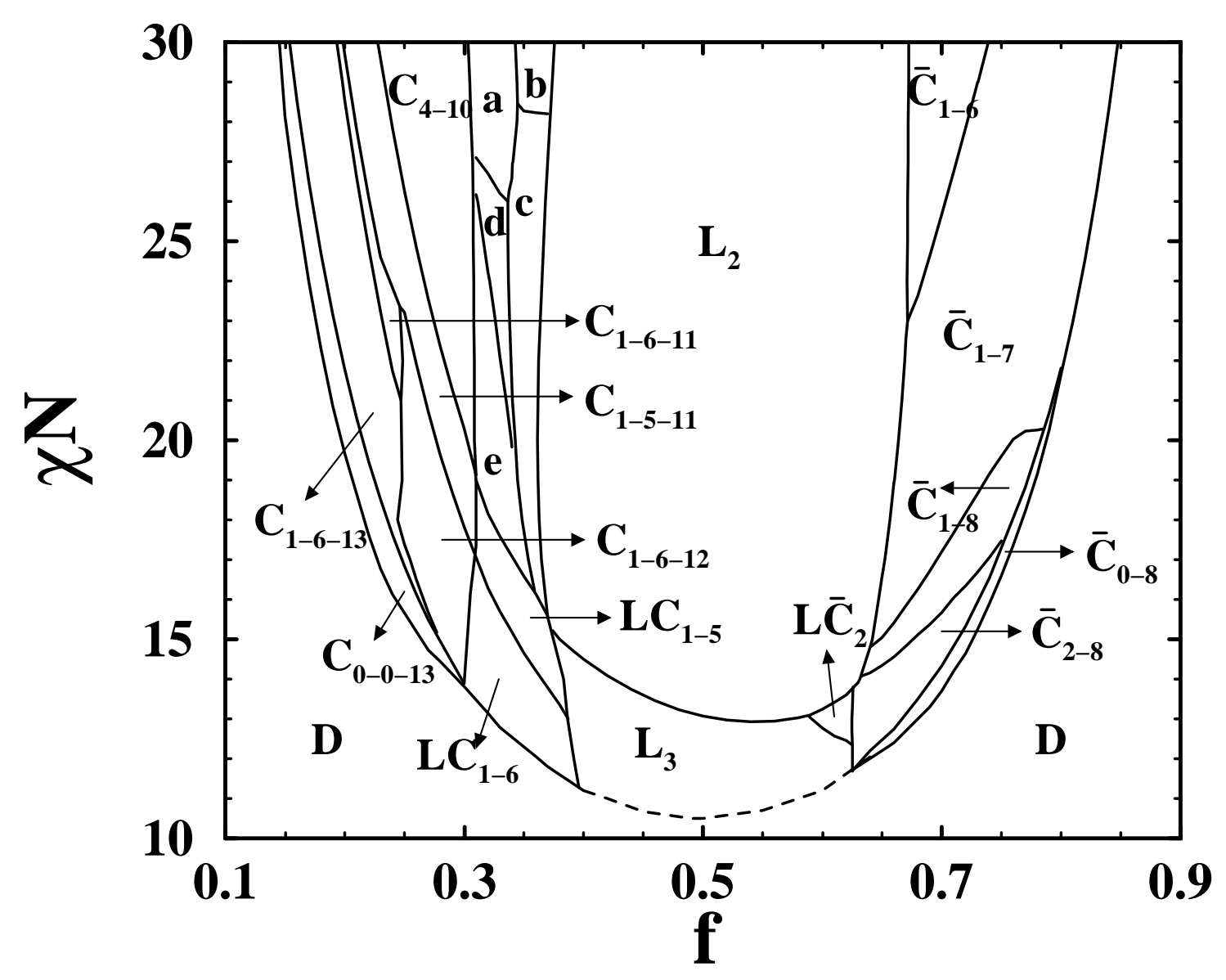



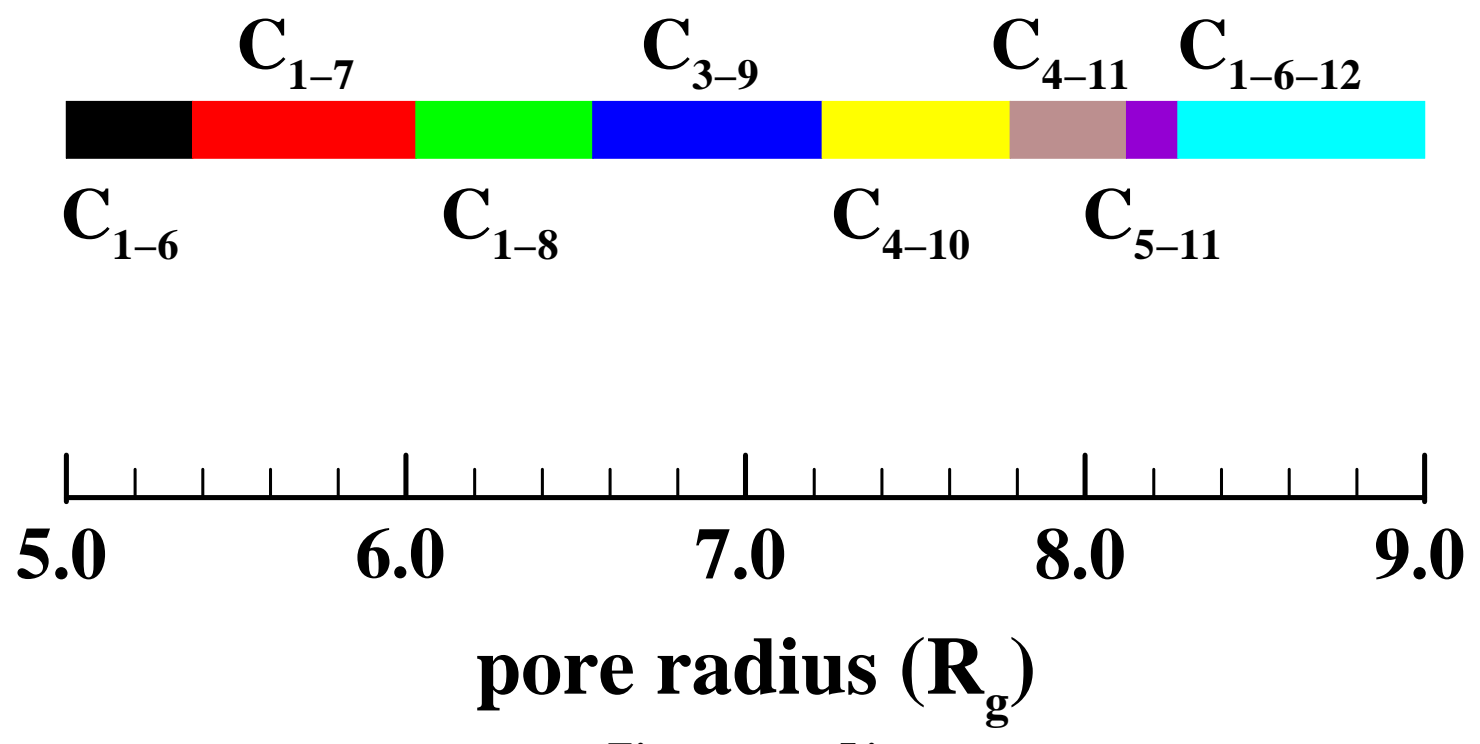

Figure $3-$ Li 